\title{
¿CADA VEZ MÁS APÁTICOS? \\ El desinterés político juvenil en España en perspectiva comparada
}

INCREASING APATHY?

Spanish youth political disinterest in comparative perspective

CAROL GaLAIS carolina.galais@uab.cat
Universitat Autònoma de Barcelona. España

ResUmen

A menudo se ha señalado que los niveles de apatía política de la juventud española son alarmantes. En estas páginas se comprueba esta afirmación con relación a la juventud europea y se intenta dilucidar si el grado de interés por la política de los jóvenes españoles ha empeorado en los últimos tiempos. Para ello se comparan las diferencias entre los jóvenes españoles y europeos con los del resto de la población, así como con los que, en España, fueron jóvenes en épocas anteriores. Finalmente, se comprueba el supuesto de la progresiva despolitización de los jóvenes mediante un análisis multivariante. Se concluye que los jóvenes actuales solo presentan unos niveles de interés por la política significativamente inferiores a los jóvenes de los años 80 , los cuales son la auténtica excepción.

\section{Palabras Clave}

Actitudes políticas; Implicación política; Jóvenes.

\begin{abstract}
It has been often claimed that the political apathy of Spanish youth is alarming. This study tests that assessment and tries to find out whether political interest among young citizens is different with respect to European youth and if it is worsening in the last years. The levels of political interest among the youth of today are compared with those of people who were young some years ago, as well as with the levels of political interest of the European youth. The hypothesis of the progressive depolitization of the youth is tested following a multivariate analysis. The article concludes that current Spanish youth is only significantly less interested if compared to the youth of the eighties, which constitutes the actual exception to the rule.
\end{abstract}

\section{KEYWORDS}

Political Attitudes; Political Engagement; Youth. 


\section{¿POR QUÉ PREOCUPARSE POR LA IMPLICACIÓN POLÍTICA DE LA JUVENTUD?}

Desde finales de la última década, los medios de comunicación han comparado en numerosas ocasiones la actitud implicada y comprometida de los jóvenes europeos con la pasividad de la juventud española, resaltando que comparten con aquellos altos niveles de paro y escasas perspectivas de mejora. En esta línea se mencionaron los ejemplos de los jóvenes franceses que en 2006 reivindicaban mejores contratos laborales, de los griegos que en 2008 paralizaron el país por el asesinato de un joven activista a manos de la policía, o de los ingleses que finalizaron 2010 protestando intensamente por el aumento de las tasas universitarias ${ }^{1}$.

No solo los medios de comunicación han enfatizado la supuesta pasividad de la juventud española respecto a la esfera pública (Alcoceba y Matheus 2010). También desde la academia se ha argumentado que nuestros jóvenes son la punta de lanza de una nueva sociedad, consentida y cortoplacista ${ }^{2}$. Así, se ha ido asentando una creencia generalizada no solo en que nuestros jóvenes destacan por su pasividad política, sino también en que esta pasividad iba en aumento en los últimos tiempos. Al menos, hasta las movilizaciones agrupadas en torno al conocido como "movimiento 15 de mayo". Pero ¿tenían estas perspectivas negativas sobre el compromiso político de la juventud española alguna base?

La mayoría de las reflexiones sobre la pasividad política de los jóvenes españoles se basa en la observación de un tipo de comportamiento político muy concreto, perteneciente al repertorio de la protesta ${ }^{3}$. Considerar también actividades como el voto o el asociacionismo no mejorará la idea que tenemos de estos jóvenes, que destacan respecto a sus mayores y también ante los jóvenes de otros países por sus altos niveles de abstención y su baja propensión a asociarse (Anduiza 2001; Morales 2003). Sin embargo, todos estos son comportamientos objetivos, dependientes no solo de la motivación de los individuos, sino también de las restricciones del contexto, los incentivos, la movilización o la disponibilidad de recursos como el tiempo, el nivel de conocimiento político y las redes sociales (Verba et al. 1995). Por este motivo, el presente trabajo intenta ir a la raíz de la cuestión de la supuesta pasividad política de la juventud, centrándose en sus

\footnotetext{
${ }^{1}$ Ver noticias al respecto en la BBC el 3 de abril de 2006 y en La Razón el 27 de noviembre de 2010. http://news.bbc.co.uk/hi/spanish/international/newsid_4873000/4873978.stm http://www.larazon.es/noticia/3918-jovenes-espanoles-por-que-no-protestan

${ }^{2}$ Algunas opiniones académicas a este respecto fueron publicadas el 19 de diciembre de 2010 en El confidencial. Ver http://www.elconfidencial.com/sociedad/grandes-movilizaciones-juveniles-europa-espanabotellon-20101218-72694.html

${ }^{3}$ Aunque no es el objeto de estudio de estas páginas, debe advertirse que, ni siquiera limitándose a un tipo de participación tan costoso como son las manifestaciones, estas afimaciones estarían en lo cierto. Diversos estudios apuntan a que los jóvenes españoles serían más críticos que pasivos respecto a la política, y que precisamente destacarían por su práctica de actividades no relacionadas con la representación política (Caínzos 2006; Morales 2005; Ferrer 2006; Jiménez 2006).
} 
bases subjetivas o psicológicas. Concretamente, se limita al análisis del interés por la política en su faceta de manifestación de curiosidad y compromiso con lo público y, por tanto, de indicador de la motivación necesaria para participar en política (Almond y Verba 1963; Verba et al. 1995; Brady 1999, y, para el caso español, Anduiza y Bosch 2004; Mateos 2004; Schreiber y García 2004; Martín 2004 y 2005; Bonet, Martín y Montero 2006; Morales, Mota y Pérez Nievas 2006).

La preocupación por una posible pasividad política de los jóvenes y su agravamiento es comprensible tanto desde el punto de vista del republicanismo cívico, que supone que los "buenos" ciudadanos se forjan en este periodo de la vida, como desde una perspectiva más pragmática que ve la política como un aspecto de la vida adulta (Morán y Benedicto 2008). En el primer caso, un seguimiento del papel de los jóvenes en la política y de su evolución puede darnos pistas de si los ciudadanos del mañana estarán más o menos comprometidos políticamente, con las consiguientes repercusiones sobre el funcionamiento y legitimidad de las instituciones representativas y sobre los valores de la sociedad. Desde la visión pragmática, la preocupación por este tema tendría el mismo sentido que prestar atención a la edad media de emancipación de la juventud: la pasividad política indicaría que no han completado su transición a la madurez, e incluso un alargamiento de esta etapa de la vida.

Al acotar la relación entre jóvenes y política únicamente a sus bases psicológicas, resulta aún más evidente por qué este objeto de estudio merece atención. Las tendencias actitudinales de los jóvenes son el motor fundamental del cambio cultural y de valores en una sociedad (Inglehart 1990). En el caso español, un aumento del compromiso, la atención y la curiosidad política supondría romper con la persistente tendencia a la apatía y al desapego político que caracterizan a la cultura política española (Gunther et al. 2004; Bonet, Martín y Montero 2006). Por el contrario, la continuidad en los niveles de interés por la política supondría la reproducción de estos patrones culturales. Finalmente, no son pocos los autores que han concluido que los jóvenes españoles de hoy en día presentan una tendencia más marcada a la apatía política que los jóvenes de antaño (Del Moral 2003; Martín 2004; González-Anleo 2005). Esto supondría la aparición en escena de una nueva cohorte de jóvenes españoles, más similar a aquellas que experimentaron la guerra civil y la postguerra —extraordinariamente ajenas a lo público— que a sus propios padres.

Existe, por tanto, una relación entre juventud e interés por la política en España que sigue sin estar clara al inicio de esta segunda década del siglo XXI. Ante ésta, cabe plantearse: ¿Están aquí los jóvenes tan poco interesados por la política como afirman las conclusiones más alarmistas sobre la despolitización de la juventud? ¿Qué implicaciones tiene el desinterés por la política de los jóvenes en España en comparación con otros países europeos? ¿Están los jóvenes españoles en los últimos años menos interesados por la política que tiempo atrás? En las siguientes páginas se tratará de responder a estas preguntas con la intención de averiguar si nos encontramos en un escenario de reproducción, superación o agravamiento de los distintos síndromes actitudinales y comportamientos políticos de los que da cuenta el interés por la política. 


\section{EL INTERÉS POR LA POLÍTICA Y EL PAPEL DE LA EDAD EN SU DESARROLLO}

El interés por la política es probablemente una de las orientaciones más generales hacia lo público. Numerosos estudios se han ocupado de esta actitud, ya sea en exclusividad o junto a otras percepciones subjetivas, midiendo fenómenos como la sofisticación política (Zaller 1992) o el apoyo político (Weatherford 1991). Así mismo, un déficit de esta actitud se ha asociado a distintos síndromes, como la alienación política (Campbell 1962), el cinismo político o la desafección política institucional (Jackman 1987; Torcal 2006).

Una lectura atenta de la pregunta más habitual con que se captura esta actitud en las encuestas de opinión pública y cultura política ("En líneas generales, ¿diría Ud. que la política le interesa mucho, bastante, poco o nada?") revela que el interés político hace referencia a la curiosidad por los asuntos relacionados con la política. Esta curiosidad condicionará la manera en que el individuo procesa la información del contexto político; es decir, es la actitud opuesta a la indiferencia (Van Deth 1990; Fiske y Taylor 1991). Desde este punto de vista, el interés equivaldría al grado en que el ciudadano está dispuesto a recibir y procesar información relacionada con la política. Tendría, por tanto, un componente cognitivo y dependería en cierta medida de las características del contexto político.

En un sentido ligeramente más amplio, el interés por la política haría referencia a su orientación hacia la política en general, al sentimiento de atracción hacia la misma (Delli Carpini y Keeter 1996). En su formulación negativa, el desapego político se considera un obstáculo para la creación de capital social, además de un impedimento para que la democracia funcione como mecanismo de control del poder político (Martín 2005). Este componente afectivo introduce en cierta manera un error de medida, puesto que en ocasiones puede estar capturando el rechazo de los ciudadanos a determinados aspectos del sistema político, y no la desatención o falta de compromiso con lo público en general. Esta limitación debe ser tenida en cuenta especialmente para el caso español, donde es frecuente manifestar sentimientos negativos (pero nada tibios, como el asco o la irritación) a la vez que escasos niveles de interés por la política (Galais 2008). Además, si esta actitud es predominantemente afectiva, se asociaría a valores y creencias simbólicas transmitidas en momentos tempranos del proceso de socialización (Pollack y Mishler 2003), lo que la haría relativamente estable a lo largo de la vida. Esto nos lleva al análisis del papel que juega la edad en el desarrollo y configuración de esta actitud.

La edad es una característica individual fundamental para el estudio de las actitudes políticas en la medida en que asigna un rol y un estatus en el sistema social (Justel 1992). En este sentido, situarse en el tramo del ciclo vital que entendemos por "juventud" predispondría a manifestar sentimientos negativos y de alineación hacia la política (Marsh, O' Toole y Jones 2007). Los jóvenes carecerían de preferencias políticas intensas por carecer de condicionantes económico-sociales que diesen forma a sus expectativas políticas, al no haber apenas entrado a formar parte de la población activa (Rosenstone y Hansen 1993). La juventud implica igualmente ausencia de responsabilidades familiares. Los jóvenes dependientes de sus padres no tendrían incentivos para 
informarse de determinadas políticas públicas que afectan a los contribuyentes (Sapiro 1994). Por otra parte, los jóvenes no han experimentado aún muchos estímulos políticos ni han entrado a formar parte de todas sus futuras redes sociales (Nie et al. 1996), lo que les haría sentirse más ajenos a los procesos políticos que los individuos adultos. Estos últimos están más integrados en la sociedad y más vinculados a las normas y restricciones propias de su situación en esta estructura (Goerres 2007). Por otra parte, también las personas mayores presentarían niveles más bajos de interés por la política debido al momento del ciclo vital en que se encuentran. A medida que sus redes sociales se deterioran, sus estímulos políticos disminuyen y su rol social se hace menos central y visible (Visser y Krosnick 1998; Durán 2007), lo que potencia su alineación respecto a la política. En definitiva, la edad guardaría una relación con el interés por la política de tipo cuadrático, con un punto de inflexión en la edad madura.

Según estos argumentos, los jóvenes presentarían -independientemente del país de origen- una escasa propensión a implicarse subjetivamente en política en comparación a los adultos, cosa que solo mejoraría al madurar. Desde otro punto de vista, no sería el ciclo vital en que se encuentran los jóvenes la causa de su apatía, sino su socialización política, lo que augura efectos negativos a largo plazo y equivale a pronosticar que su desapego político no mejorará con la madurez. Los jóvenes actuales serían los responsables de una disminución en las actitudes cívicas de la ciudadanía (Putnam 1995; 2000 y 2002). Sin embargo, no existen muchos motivos para pensar que los valores, educación y experiencias tempranas del sistema político que caracterizan la socialización de los jóvenes españoles son políticamente alienantes. Por el contrario, estamos ante una generación de españoles con un nivel de educación más elevado que el de sus mayores. Han crecido en un período de normalidad democrática, dominan nuevos repertorios de acción y movilización social que reducen enormemente los costes de la acción colectiva y no se ha alentado su desmovilización política desde las instituciones como ocurrió durante el franquismo.

Aunque no existan razones de peso para creer que los jóvenes españoles están bajo los efectos de una socialización política alienante, podríamos encontrar en este hilo de pensamiento el origen de la creencia en una creciente apatía juvenil. Y es que este grupo de edad está siendo implícitamente comparado con una generación, los actuales adultos maduros que rondan los cincuenta años, cuyos niveles de interés y compromiso político fueron excepcionales. Dicho de otro modo, los jóvenes de hoy no podrían competir con la intensa politización de los que eran jóvenes cuando empezamos a disponer de datos de opinión pública y cultura política fiables provenientes de encuestas españolas, a principios de los 80 . Los que entonces tenían menos de 30 años crecieron durante la última fase del franquismo y la fase más movilizadora de la Transición, un contexto capaz de forjar no solo sólidas lealtades hacia el sistema democrático, sino también sentimientos de competencia y compromiso políticos intensos y duraderos (Montero et al. 1998).

Dicho contexto es irrepetible y aquella juventud extraordinariamente atenta y comprometida fue un fenómeno único y extraño, porque contradice los efectos esperados del ciclo vital sobre el interés político respecto a los que la literatura ha llegado a un cierto 
consenso. Si este argumento está en lo cierto, la relación entre interés por la política y edad debería haber evolucionado a lo largo del tiempo desde una función lineal y negativa (asociando juventud a altos niveles de interés) a una de tipo cuadrático, a medida que aquella generación más implicada ha ido envejeciendo.

Recapitulando lo expuesto hasta ahora, existe una creencia cada vez más afianzada en el progresivo desinterés por la política de la juventud española. Sostener que los jóvenes españoles son cada vez más ajenos a la política equivale a afirmar que el efecto del ciclo vital sobre el interés por la política ha aumentado su intensidad en los últimos años; que para el caso español no se aplica la teoría que predice un efecto cuadrático de la edad sobre esta actitud, o que estamos asistiendo a la aparición de una cohorte que destaca por su apatía política. Estos extremos pueden comprobarse empíricamente mediante la comparación de los niveles de interés por la política de los jóvenes españoles con los de otros países y con los que manifestaron los jóvenes en España en épocas anteriores. Los objetivos fundamentales de este trabajo son analizar la relación que guardan edad e interés por la política y comprobar si los jóvenes españoles están cada vez menos interesados en política, teniendo en cuenta el último período histórico, o de normalidad democrática. Se intentará demostrar que la actual juventud española no es excepcionalmente apática si la comparamos con sus mayores y que su actitud hacia la política es susceptible de variación - como defienden los autores que enfatizan su componente cognitivo- porque depende en gran medida del contexto político, operacionalizado a partir del año en que se midió dicha actitud.

\section{¿SON LOS JóVENES ESPAÑOLES REALMENTE TAN APÁtICOS RESPECTO A LA POLÍtICA? DATOS Y RESULTADOS}

En los siguientes análisis se considerarán "jóvenes" aquellas personas de hasta 30 $a^{n}{ }^{4}{ }^{4}$. Para comprobar las expectativas planteadas hasta ahora se han elegido la cuarta ola de la Encuesta Social Europea (ESE), llevada a cabo entre 2008 y 2009 en 28 países, así como 11 encuestas representativas de la población española realizadas por el Centro de Investigaciones Sociológicas (CIS) entre 1985 y 2008 en que se pregunta por el interés por la política.

En primer lugar, mediante la encuesta ESE se analizará el impacto de la carencia de esta actitud política (esto es, del desinterés) sobre distintos tipos de participación

\footnotetext{
${ }^{4}$ Para una discusión breve y muy pertinente de los límites de la juventud, ver García y Martín (2010). Las autoras señalan que el límite más generoso de la juventud está puesto en los 34 años y que la frontera más comúnmente aceptada en ciencia son los 30 años. Un motivo adicional para situar la frontera de la juventud en los 30 años pese a que la tendencia actual es ampliar paulatinamente los límites de este grupo etario, es que en estas páginas tenemos en consideración a los que fueron jóvenes a principios y mediados de los 80 , cuando muy difícilmente podía argumentarse que una persona de más de 30 años no era totalmente adulta.
} 
para los países participantes en esta ola del estudio que formaron parte de la UE15, con la finalidad de entender su alcance y repercusiones sobre la implicación política. A continuación, se compararán los niveles de interés político de los jóvenes españoles con los de estos países europeos, con la finalidad de averiguar si realmente el caso español es excepcional ${ }^{5}$. Finalmente, se estimarán los valores de interés por la política a partir del efecto cuadrático de la edad tanto en los once países europeos seleccionados como en los once años para los que tenemos datos del caso español. De esta manera se comprobará hasta qué punto es habitual encontrar una relación de tipo no lineal entre estas dos variables en distintas sociedades y en qué medida dicha relación puede evolucionar a lo largo del tiempo.

En cuanto a las encuestas del CIS, se ha puesto cuidado en que reflejen el período más reciente posible (2004 en adelante) para poder realizar un seguimiento de la juventud actual. A partir de las mismas se comprobará la hipótesis nula de la creciente apatía política juvenil estimando el interés por la política a partir de la edad, el año de medición de esta actitud y las interacciones entre el hecho de ser joven y los distintos años incluidos en el análisis, que deberían resultar significativos si la juventud realmente es cada vez más ajena a la política.

Con el objetivo de analizar el alcance de la escasez o ausencia de esta actitud sobre la participación política, la tabla 1 presenta los resultados de distintas estimaciones logísticas para seis tipos distintos de actividad política en los once países europeos participantes en la cuarta ola de la ESE miembros de la UE15. Estos datos únicamente hacen referencia a la población de entre 18 y 30 años de los mencionados países. Los datos son razones de probabilidades (odds ratios) relativas al efecto de estar poco o nada interesados por la política (frente a estarlo bastante o mucho), teniendo en cuenta también el género y los años de educación.

El primer dato que salta a la vista es que todas las razones de probabilidades son inferiores a 1, lo que confirma a esta actitud política como buen predictor de la participación, puesto que unos bajos niveles de interés se relacionan con la pasividad política. Sin embargo, el alcance de este efecto no es homogéneo entre países. En algunos, estar desinteresado no comprometería la acción política o apenas la perjudica. Este sería el caso de Bélgica, donde manifestarse desinteresado por la política solo tiene un efecto negativo y significativo sobre el boicot y el trabajo en organizaciones. En Finlandia, Alemania o Francia, estos valores se acercan más al uno, indicando que los ciudadanos que manifiestan desinterés por la política no son tan reacios a participar en ella como en otros países, o en otras palabras, que apenas existen diferencias entre el hecho de estar interesado y no estarlo para acabar tomando parte en estas actividades.

${ }^{5}$ Aunque en la ESE se incluyen individuos desde los 15 años, se han seleccionado únicamente a los que tenían entre 18 y 90 años (ambos inclusive) en el momento en que fueron encuestados. De esta manera, las conclusiones sobre la "juventud" española serán comparables con las que se deriven del uso de las encuestas CIS, que únicamente incluyen individuos mayores de edad. 
Tabla 1.

Estimación de distintos tipos de participación a partir del desinterés político de los jóvenes europeos.

\begin{tabular}{lcccccc}
\hline & Voto & $\begin{array}{c}\text { Trabajo en } \\
\text { organizaciones }\end{array}$ & $\begin{array}{c}\text { Firma de } \\
\text { peticiones }\end{array}$ & Contacto & Boicot & Manifestaciones \\
\hline Bélgica & Ns. & $0,4^{* *}$ & Ns. & Ns. & $0,52^{\dagger}$ & Ns. \\
Alemania & $0,36^{* *}$ & $0,63^{*}$ & $0,49^{* *}$ & Ns. & $0,58^{*}$ & $0,38^{* *}$ \\
Dinamarca & Ns. & $0,4^{*}$ & $0,38^{* *}$ & $0,31^{\dagger}$ & $0,3^{* *}$ & $0,22^{* *}$ \\
España & $0,23^{* *}$ & $0,21^{* *}$ & $0,39^{* *}$ & $0,11^{* *}$ & $0,34^{* *}$ & $0,27^{* *}$ \\
Finlandia & $0,57^{* *}$ & $0,54^{* *}$ & $0,42^{* *}$ & $0,38^{* *}$ & $0,58^{*}$ & Ns. \\
Francia & Ns. & $0,22^{* *}$ & $0,36^{* *}$ & $0,54^{\dagger}$ & $0,61^{\dagger}$ & $0,41^{* *}$ \\
Reino Unido & $0,49^{* *}$ & $0,20^{* *}$ & $0,36^{* *}$ & Ns. & $0,36^{* *}$ & $0,21^{*}$ \\
Grecia & Ns. & $0,15^{* *}$ & $0,38^{*}$ & $0,18^{* *}$ & $0,57^{\dagger}$ & $0,11^{* *}$ \\
Países Bajos & $0,43^{*}$ & $0,47^{*}$ & $0,49^{*}$ & $N s$. & $0,21^{* *}$ & $N s$. \\
Portugal & $0,35^{* *}$ & $0,36^{\dagger}$ & $0,29^{* *}$ & $0,27^{* *}$ & $N s$. & $0,27^{* *}$ \\
Suecia & $0,43^{*}$ & $0,62^{\dagger}$ & Ns. & $0,21^{* *}$ & $0,45^{* *}$ & $0,16^{* *}$ \\
\hline
\end{tabular}

$t=p<0,1 ;{ }^{*}=p<0.5 ;{ }^{* *}=p<0.01$.

Odds ratios de las estimaciones de distintos tipos de actividad política a partir del desinterés político. Controles: género y educación.

La población analizada se limita a jóvenes de entre 18 y 30 años, ambos inclusive. Datos de la cuarta ola de la ESE (2008-2009). Se han aplicado las ponderaciones necesarias para trabajar con muestras representativas de cada país analizado.

En España, en cambio, el desinterés juvenil se asociaría a sentimientos negativos orientados a actores e instituciones de la representación política, motivo por el que este rasgo perjudica especialmente a las actividades de contacto con actores políticos. En Grecia, Reino Unido o Suecia, esta actitud podría hacer referencia más bien a los asuntos públicos en general, afectando a la motivación necesaria para informarse sobre estos temas, y perjudicando la propensión a realizar actividades más costosas en términos de riesgo o tiempo invertido, como las manifestaciones, el contacto o el trabajo en organizaciones. Todo ello indica que, aunque siempre relacionada con la participación política, esta actitud puede tener distintas connotaciones en distintos contextos, orientándola más a procesos, instituciones o actores y afectando con más o menos intensidad a los distintos tipos de participación. Pero ¿puede ser que estas distintas connotaciones en función del contexto afecten también a la relación entre interés por la política y edad? 
Figura 1.

Proporción de jóvenes interesados por la política. ESE 2008-2009.

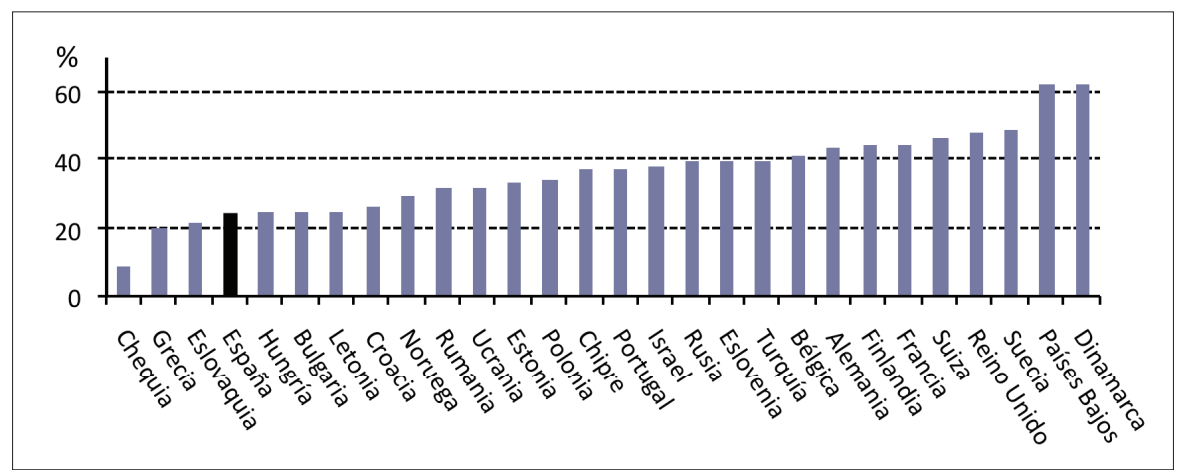

Fuente: Elaboración propia a partir de la cuarta ola de la ESE.

La Figura 1 muestra los porcentajes de ciudadanos de hasta 30 años que manifiestan mucho 0 bastante interés por la política para los países participantes en la cuarta ola de la ESE. España es el cuarto país con una proporción más baja de jóvenes interesados por la política; solo por delante de Eslovaquia, Grecia y Chequia. Por el contrario, países como Dinamarca, Países Bajos o Suecia destacan por la elevada proporción de ciudadanos interesados entre el sector más joven de la población. Así, la excepcionalidad de los niveles de desinterés político entre la juventud española se confirma en perspectiva comparada. Sin embargo, si la población joven no está más desinteresada que los adultos y ancianos podríamos estar únicamente ante una de las facetas de una desafección generalizada por la política en algunos países, España entre ellos, un fenómeno relacionado a su vez con la longevidad de las instituciones democráticas y el nivel de desarrollo económico (Torcal 2006).

Con la finalidad de abundar en los detalles de la relación entre edad e interés por la política y saber en qué medida se ajusta en distintos países a la relación cuadrática que la literatura predice, se ha estimado esta última variable únicamente a partir del efecto cuadrático de la edad mediante una regresión de mínimos cuadrados ${ }^{6}$. Para ello, se ha recodificado la variable interés de manera que sus valores estén comprendidos entre 0 y 1. Los valores predichos para estas estimaciones se presentan de manera gráfica en la figura 2, donde los países aparecen ordenados en función de la capacidad predictiva de este modelo, es decir, por el valor de la R cuadrado. Así, mientras que en Finlandia

${ }^{6}$ Los valores predichos se han calculado a partir de la ecuación Interés por la política $=\mathrm{B} 0+\mathrm{B} 1{ }^{\text {* }}$ edad + $\mathrm{B} 2 \mathrm{edad}^{2}+\mathrm{e}$. 
este valor es cercano a 0 (indicando que esta variable no explica variación alguna del interés por la política), en Portugal el efecto cuadrático de la edad predice hasta un 1,2\% de variación del fenómeno analizado. En España este modelo apenas consigue predecir un $0,3 \%$ de la variación del interés, una situación similar a la de Bélgica o Países Bajos, debida a las escasas diferencias entre grupos de edad.

En líneas generales, es habitual encontrar la relación cuadrática que la literatura predice, aunque la misma es más clara en Alemania, Reino Unido o Países Bajos. España no sería una excepción, aunque en su caso, como en Francia y a diferencia de lo que sucede en Grecia o Bélgica, las diferencias entre generaciones son menores y los más jóvenes presentan valores predichos de interés por la política ligeramente más bajos que el resto de la población. Esta última tendencia es, sin embargo, mucho más acusada en Portugal. En conclusión, la relación cuadrática entre edad e interés por la política presenta variaciones notables entre países. El caso español se ajustaría a esta teoría -aunque con menores diferencias entre grupos de edad y una juventud ligeramente más pasiva que en

Figura 2.

Interés político predicho por la edad (Cuarta ola ESE 2008-2009).

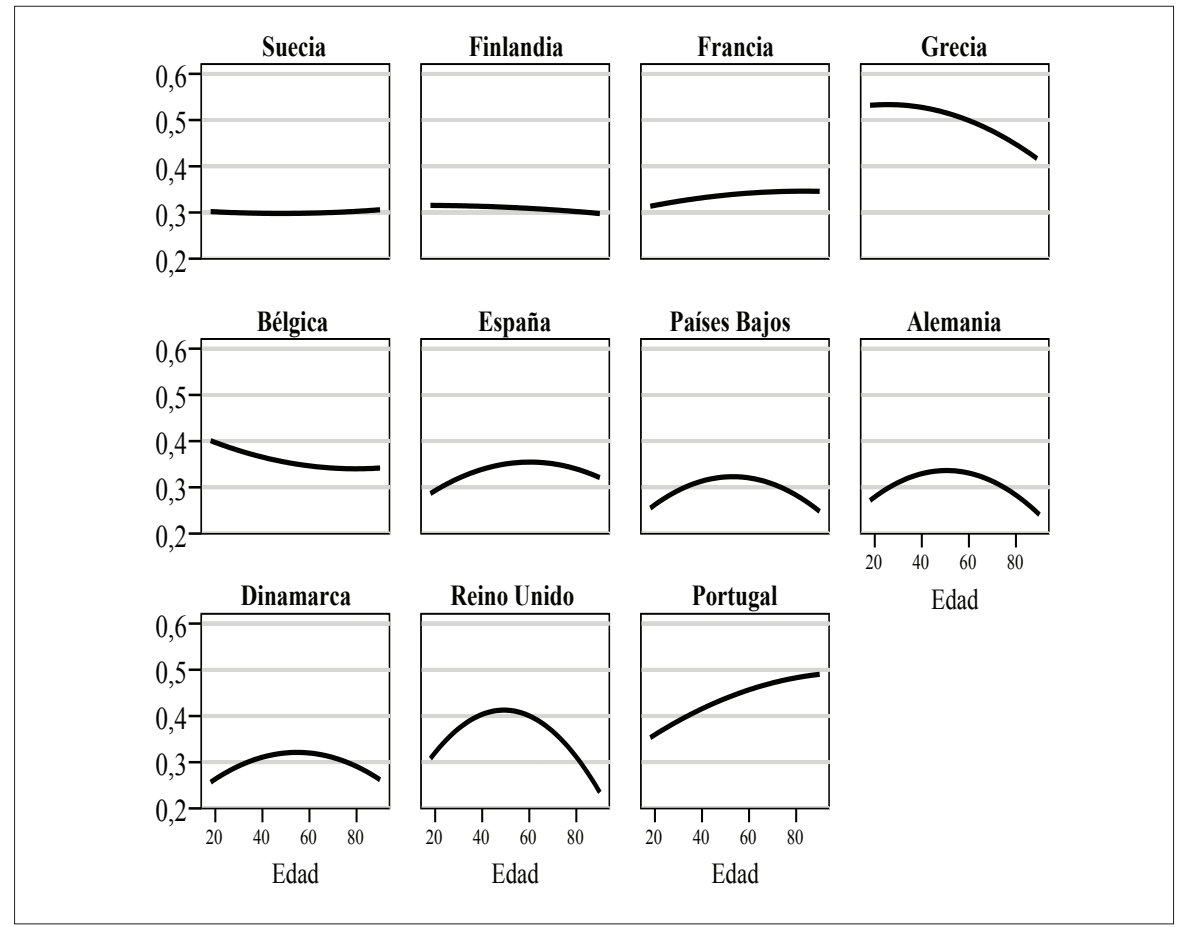

Fuente: Elaboración propia a partir de la cuarta ola de la ESE.

Número de casos válidos: Bélgica, 1681; Alemania, 2658; Dinamarca, 1550; España, 2480; Finlandia, 2105; Francia, 1973; Reino Unido, 2240; Grecia, 1996; Países Bajos, 1714; Portugal, 2263; Suecia, 1745. 
otros países-, a diferencia de Portugal o Grecia, dos sociedades donde dicha relación es más lineal —-positiva y negativa, respectivamente- que curvilínea.

De igual manera, cabe preguntarse si la relación encontrada para el caso español es estable a lo largo del tiempo o bien si se trata de un fenómeno reciente. Con esta finalidad, y de manera similar a como se procedió para obtener los datos representados en la figura 2, se ha estimado el interés por la política únicamente a partir del efecto cuadrático de la edad para once años comprendidos entre 1985 y 2008. Para ello, se han fusionado en una misma base de datos la información procedente de once encuestas del CIS realizadas entre 1985 y 2008, trabajando con los datos como si fueran un falso panel y respetando las ponderaciones indicadas en cada encuesta para obtener muestras representativas de la población española. Los datos, presentados en la figura 3, revelan que al inicio del período existe una relación casi lineal y negativa entre edad e interés: los más jóvenes están más interesados, como ocurría en 2008-2009 para Grecia o Bélgica. Esta tendencia se transforma paulatinamente en la relación cuadrática que la literatura

Figura 3.

Valores del interés por la política predichos por la edad. España: 1985-2008.

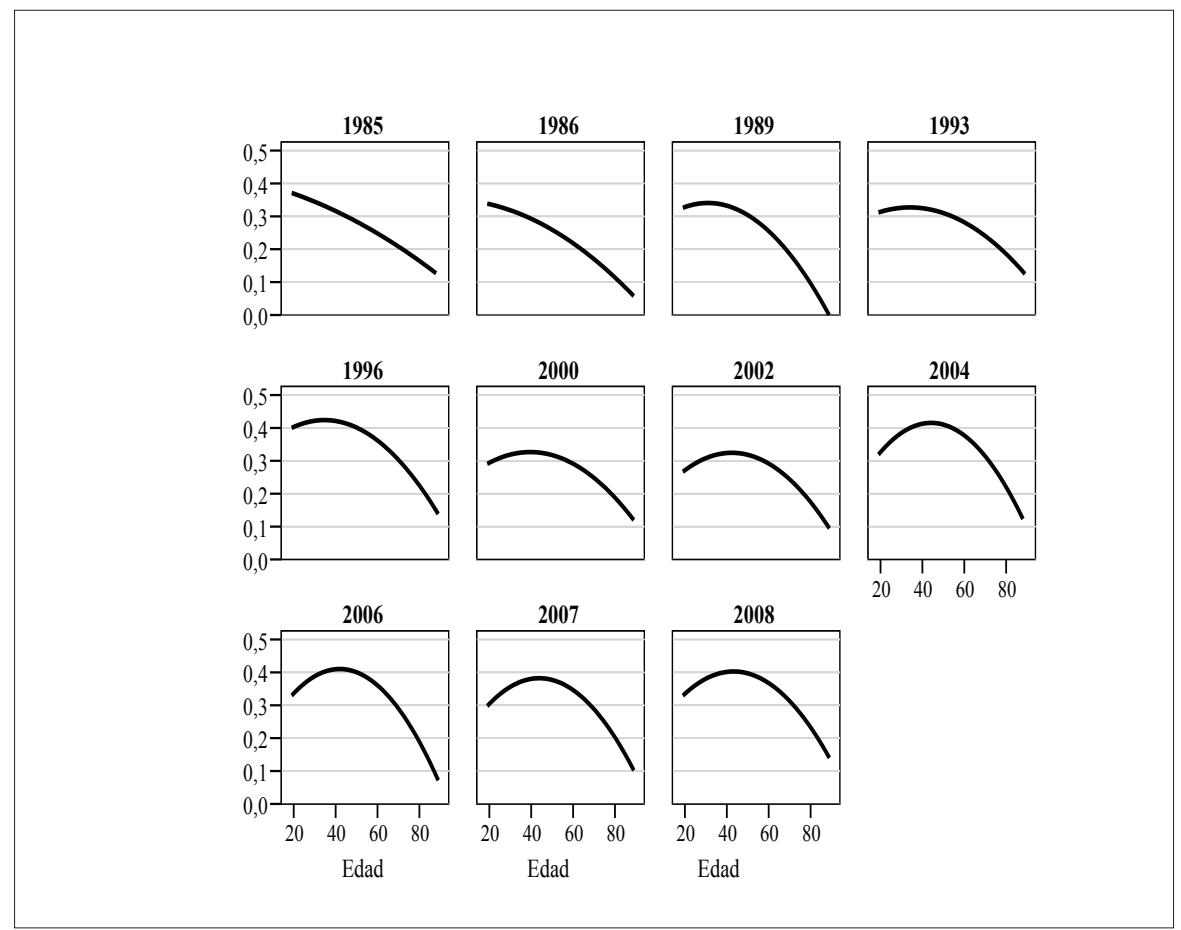

Fuente: elaboración propia a partir de estudios CIS 1461 (1985), 1788 (1989), 2206 (1996), 2450 (2002), 2535 (2003), 2575 (2004), 2620 (2005), 2632 (2006), 2736 (2007) y 2760 (2008). 
predice a mediados de los años noventa, y ya claramente a partir del año 2000 , cuando los jóvenes de los años 80 han ingresado ya todos en el grupo de edad madura y las diferencias entre jóvenes, ancianos y adultos disminuyen. Aún así, es destacable que los valores predichos del interés por la política del segmento de edad más joven son más elevados en 1996 y a partir de 2004 que en los años 80.

Así, el progresivo desinterés por la política de los jóvenes españoles parece ser un mito, al menos por lo que respecta al análisis de los datos aquí expuestos. Más bien parece que dicha relación esté adaptándose a la mencionada función cuadrática, evolucionando desde una situación similar a la que encontramos en 2008-2009 en Grecia hacia la que encontramos en Reino Unido, Países Bajos o Alemania. Desde este punto de vista, todas las situaciones en que no encontramos la típica relación cuadrática sugerirían la presencia de una cohorte, bien de adultos 0 ancianos (relación lineal y positiva entre edad e interés) o bien de jóvenes (relación negativa entre edad e interés) que rompe con esta tendencia; aunque no puede descartarse que en los países en que se cumple la relación curvilínea mencionada estén solapándose a la vez los efectos del ciclo vital con los de una cohorte de mediana edad más interesada por la política (véase el caso de España). Por otra parte, para el caso español son notables las oscilaciones en los niveles de interés por la política entre años. Las mismas pueden deberse a las características de la coyuntura política en que el ciudadano se desenvuelve. Aunque estos efectos no sean abordados sistemáticamente en estas páginas, cabe tener presente la gran sensibilidad de los jóvenes a las circunstancias y la coyuntura política que los rodea (Plutzer 2002; Franklin 2003 y 2004).

\section{ANÁLISIS MULTIVARIANTE: EL EFECTO DE LA EDAD SOBRE EL INTERÉS POR LA POLÍTICA DE LOS ESPAÑOLES EN LAS ÚLTIMAS DOS DÉCADAS}

Finalmente, se ha estimado esta actitud a partir de una regresión ordinal en que se ha incluido, además del efecto de la juventud, el sexo y el nivel de estudios como controles ${ }^{7}$. Además de tomar en consideración las ponderaciones indicadas en cada encuesta, las estimaciones se han realizado teniendo en cuenta errores estándar robustos agrupados por año. Los resultados se presentan en la tabla 2.

\footnotetext{
${ }^{7}$ Las categorías de la variable dependiente vuelven a ser en este caso "ninguno" (0), "poco" (1), "bastante" (2) y "mucho" (3). El nivel de estudios toma distintos valores y número de categorías a lo largo de los años, por lo que se codificó como una variable ordinal de tres categorías. El valor "nivel bajo" agrupa todas las posibilidades hasta estudios primarios completados, lo que incluye, entre otros, las personas sin estudios o con estudios primarios no completados. El nivel de estudios alto recoge a todos los que han pasado por la universidad para realizar estudios de cualquier duración, así como a los postgraduados. El nivel de estudios medio recoge a las personas que han superado el nivel de estudios primario pero no han llegado a cursar estudios universitarios (estudios de grado medio, Formación Profesional, bachillerato, etc.).
} 
En esta estimación se tiene en cuenta la edad codificada como una variable ordinal de tres categorías: jóvenes (mayores de edad pero menores de 31 años), adultos (mayores de 30 años y menores de 60, categoría de referencia en este análisis) y personas mayores

Tabla 2.

Estimación de los efectos de la juventud sobre el interés por la política.

\begin{tabular}{|c|c|c|c|}
\hline & Coef. & $\begin{array}{l}\text { Errores típicos } \\
\text { robustos }\end{array}$ & Sig. \\
\hline Hombre & 0,517 & 0,024 & 0,000 \\
\hline Nivel de estudios medio & 0,837 & 0,028 & 0,000 \\
\hline Nivel de estudios alto & 1,634 & 0,037 & 0,000 \\
\hline Joven (18 a 30 años) & $-0,272$ & 0,088 & 0,000 \\
\hline Mayores (60 años o más) & $-0,316$ & 0,040 & 0,000 \\
\hline Año 1985 & $-0,278$ & 0,021 & 0,000 \\
\hline Año 1986 & $-0,518$ & 0,022 & 0,000 \\
\hline Año 1989 & $-0,273$ & 0,011 & 0,000 \\
\hline Año 1993 & $-0,167$ & 0,021 & 0,000 \\
\hline Año 1996 & 0,413 & 0,020 & 0,000 \\
\hline Año 2000 & $-0,322$ & 0,021 & 0,000 \\
\hline Año 2002 & $-0,249$ & 0,020 & 0,000 \\
\hline Año 2004 & 0,163 & 0,019 & 0,000 \\
\hline Año 2006 & $-0,257$ & 0,021 & 0,000 \\
\hline Año 2007 & $-0,139$ & 0,019 & 0,000 \\
\hline Joven * 1985 & 0,335 & 0,090 & 0,000 \\
\hline Joven * 1986 & 0,404 & 0,091 & 0,000 \\
\hline Joven * 1989 & - & - & - \\
\hline Joven * 1993 & $-0,077$ & 0,091 & 0,399 \\
\hline Joven * 1996 & 0,013 & 0,091 & 0,889 \\
\hline Joven * 2000 & $-0,220$ & 0,093 & 0,018 \\
\hline Joven * 2002 & $-0,186$ & 0,091 & 0,041 \\
\hline Joven * 2004 & $-0,296$ & 0,092 & 0,001 \\
\hline Joven * 2006 & $-0,092$ & 0,092 & 0,315 \\
\hline Joven * 2007 & $-0,082$ & 0,091 & 0,369 \\
\hline /cut1 & $-0,261$ & 0,034 & 0,000 \\
\hline /cut2 & 1,548 & 0,025 & 0,000 \\
\hline /cut3 & 3,668 & 0,057 & 0,022 \\
\hline $\mathrm{N}$ & 54.001 & & \\
\hline R2 de Nagelkerke & 0,142 & & \\
\hline
\end{tabular}

Estimación mediante regresión ordinal logística. Nivel de estudios, categoría de referencia = "bajo": sin estudios, primarios completados 0 no, enseñanza secundaria obligatoria. Edad, categoría de referencia $=$ "adultos" (31 a 59 años). Año, categoría de referencia $=2008$. Interacción de referencia $=$ joven * 2008. La interacción entre 1989 y la variable "joven" fue excluida automáticamente del modelo debido a la multicolinealidad que provocaba. 
Figura 4.

Probabilidades predichas de la categoría "ningún interés por la política" para hombres con nivel de estudios medio.

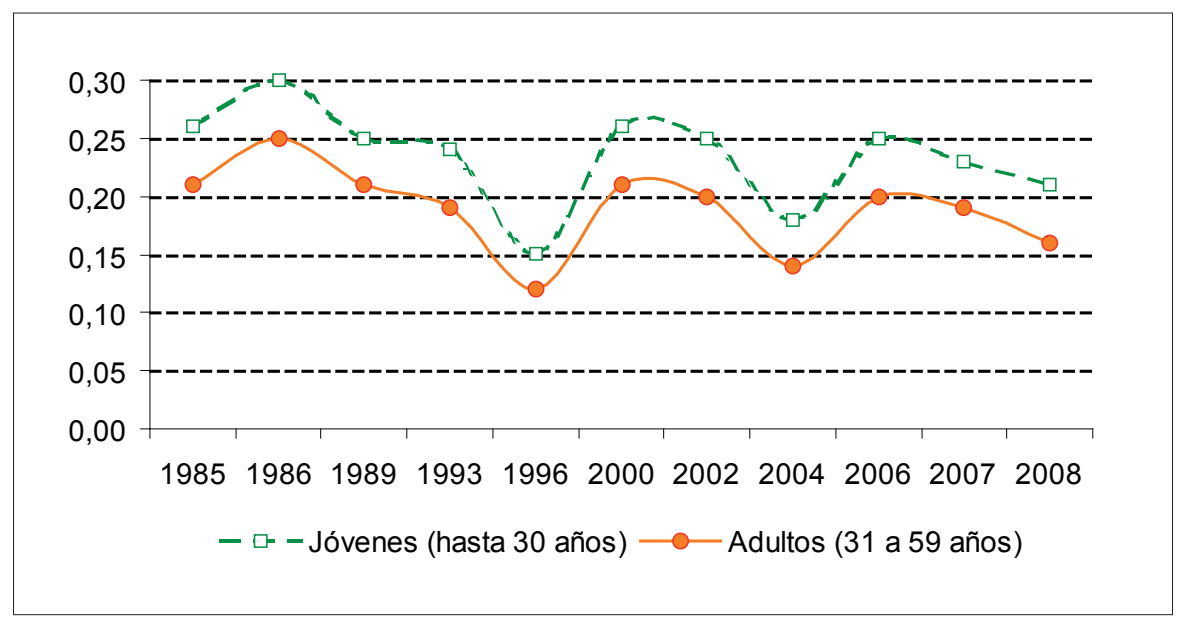

Fuente: Elaboración propia a partir de estudios CIS 1461 (1985), 1788 (1989), 2206 (1996), 2450 (2002), 2535 (2003), 2575 (2004), 2620 (2005), 2632 (2006), 2736 (2007) y 2760 (2008). Probabilidades calculadas a partir de la estimación presentada en la Tabla 2.

(60 años o más). Los resultados se controlan también por el año en que se realizaron las diferentes encuestas utilizadas, tomando como referencia el último año de la serie (2008). Se ha calculado asimismo el efecto de la interacción entre la variable dicotómica "joven" y los distintos años. Estas interacciones tratan de capturar el efecto de ser joven en esos años comparándolo con el hecho de ser joven en el año de referencia (2008).

Los efectos principales de estas interacciones indican que ser joven en 2008 tiene un efecto significativo y negativo, como apunta el coeficiente de la variable "joven" $(-0,272)$. Los coeficientes para cada año apuntan al impacto de tener 30 años o más en cada uno de esos años en comparación con 2008; y en todos los casos ese efecto es negativo y significativo salvo en 1996 y 2004, indicando un posible efecto positivo del contexto sobre este grupo de edad, ya que las elecciones de 1996 y 2004 son excepcionales tanto por sus niveles de competitividad electoral como por ser elecciones de cambio de ciclo ${ }^{8}$. Tal vez el contexto que acompañó a las elecciones en estos dos años evocó algunas de las condiciones de la Transición a las personas de edad madura, recordándoles su juventud. El caso es que, con

\footnotetext{
${ }^{8}$ Obviamente en el caso de 2004 las elecciones se celebran en un clima también excepcional y bajo el trauma reciente de los atentados del $11 \mathrm{M}$ y de su gestión por parte del Gobierno.
} 
Figura 5.

Probabilidades predichas de la categoría "mucho interés por la política" para hombres con nivel de estudios medio.

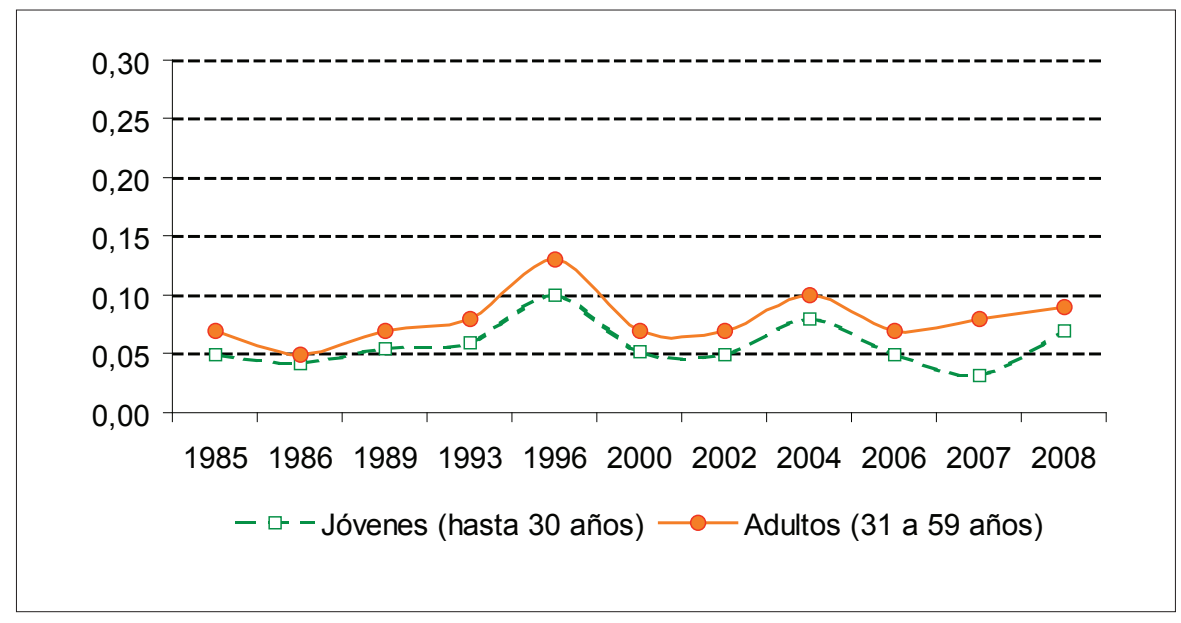

Fuente: Elaboración propia a partir de estudios CIS 1461 (1985), 1788 (1989), 2206 (1996), 2450 (2002), 2535 (2003), 2575 (2004), 2620 (2005), 2632 (2006), 2736 (2007) y 2760 (2008). Probabilidades calculadas a partir de la estimación presentada en la tabla 2.

la excepción de estos dos años, los españoles mayores de 30 años son menos propensos a manifestar niveles elevados de interés por la políica que los jóvenes en 2008.

Centrándonos ya en los efectos de las interacciones, cabe fijarse en que únicamente resultan significativas y presentan signo positivo las relativas al hecho de ser joven en 1985 y 1986. Esto significa que la juventud solo tiene un efecto significativamente mayor al del último año de la serie en dichos años. En el resto, los jóvenes presentan unos niveles de interés por la política menores que en 2008 (2000, 2002, 2004) o no significativamente diferentes (1989, 1993, 1996, 2006, 2007). Conviene señalar, sin embargo, que esta evolución no es monotónica. La tendencia no se ha ido invirtiendo paulatinamente partiendo de unos jóvenes muy implicados en los ochenta y acabando con unos jóvenes alienados en 2008, sino que varía claramente de año a año, una vez que controlamos por el efecto del sexo y el nivel de estudios.

A continuación, se muestran las probabilidades predichas por esta estimación para los hombres con un nivel de estudios medio y que pertenecen a los grupos de edad de hasta 30 años y entre 31 y $59^{9}$. Presentamos únicamente los resultados para las

\footnotetext{
${ }^{9}$ Aunque se han calculado, no se presentan las probabilidades predichas para el grupo de personas
} 
categorías "ningún interés" y "mucho interés" por la política. En la figura 4 se observa que las probabilidades de no estar interesado por los asuntos públicos no evolucionan con una tendencia clara a la baja 0 al alza a lo largo del tiempo para ninguno de los dos grupos de edad, y tampoco se aprecia que ambos grupos se distancien cada vez más, aunque en algunos años (como 1989, 1996 o 2004) llegaron a estar ligeramente más próximos e igualados en sus probabilidades de estar políticamente alienados.

En la figura 5 se presentan las probabilidades predichas de estar muy interesado por la política para los hombres con nivel de estudios medio. En este caso se observa que las probabilidades de los dos grupos de edad se distancian en algunos años, como en 1996 $y$, especialmente, en 2007. Sin embargo, ambos grupos no se alejan irreversiblemente desde entonces. La probabilidad de que un joven se interese mucho por la política se recupera al año siguiente, casi igualando las probabilidades de un adulto con el mismo sexo y nivel de estudios.

\section{DISCUSIÓN Y CONCLUSIONES}

En estas páginas se ha presentado la relación negativa entre juventud e interés por la política para una serie de democracias europeas, en que el caso español constituye una excepción porque presenta una proporción muy escasa de jóvenes muy o bastante interesados por la política. Esto es preocupante en la medida en que la ausencia de esta orientación política predice probabilidades más bajas de realizar actividades políticas más incluso en España que en otros países europeos, y especialmente si las actividades suponen dirigirse a representantes políticos. Sin embargo, el hecho de declararse desinteresado no se relaciona tan negativamente con la aversión a las manifestaciones en España como en Dinamarca, Reino Unido, Grecia o Suecia. Algo similar puede decirse de la firma de peticiones 0 el boicot, donde en otros países es menos probable que se lleve a cabo por jóvenes desinteresados que en España. Ello indica que el componente afectivo de esta actitud en España lleva a muchas personas a manifestar desinterés cuando en realidad sí se orientan con atención y compromiso a la esfera pública.

También se ha observado que la relación cuadrática entre edad e interés político no es universal, aunque sí se da en el caso español, que destaca porque sus jóvenes se parecen bastante a sus mayores respecto a su escasa tendencia a interesarse por la política. En definitiva, la relación entre desinterés político y juventud es mucho menos intensa en España que en otros países europeos.

mayores (60 o más años) porque se solapan casi perfectamente con los jóvenes y dificultan la interpretación de los gráficos. 
Por otra parte, no parece ser cierto que los jóvenes de ahora estén menos interesados por la política que sus antecesores. Una vez controlado el efecto del hecho de hacerse mayor por el del sexo, el nivel de estudios y el año en que se midió el interés por la política, puede descartarse que madurar sea, en el último período histórico, un fenómeno que potencie cada año más el interés por la política 0 , desde otro punto de vista, que ser joven sea una condición que perjudique al interés político más intensamente a medida que pasan los años. Sí puede decirse, sin embargo, que la relación lineal y positiva que encontramos entre el interés por la política y la edad a principios de los 80 se transforma paulatinamente en una relación curvilínea, tal y como predice la literatura sobre los efectos del ciclo vital en las actitudes cívicas.

La comparación de los jóvenes actuales con los que protagonizaron la Transición a la democracia y los que vivieron la llegada al poder del Partido Socialista en 1982 -en las que han sido hasta la fecha las elecciones generales con mayor participación- no beneficia a los primeros. Los que fueron jóvenes hasta mediados de los años ochenta eran seguramente una excepción, igual que las circunstancias en las que transcurrieron los años impresionables de su socialización política. Lo normal, según la literatura relativa a los efectos de la edad sobre las actitudes cívicas, es que los jóvenes se asemejen a los ancianos y destaquen por su apatía. Como demuestran los datos europeos analizados en estas páginas, el efecto del ciclo vital es generalmente negativo para estos dos grupos de edad a menos que algo excepcional, como una nueva cohorte más comprometida y apasionada por la política, aparezca en escena. Podríamos aventurar incluso que los países en que la relación entre ciclo vital e interés por la política no es claramente curvilínea (Grecia, Bélgica o Portugal) acusan un efecto cohorte entre los más jóvenes o los más viejos que rompe con esta tendencia.

Los actuales jóvenes españoles no configuran una generación excepcional que despunte por arriba, como sus padres, pero tampoco por abajo, como sus abuelos y bisabuelos. Sin embargo, algo en su socialización ha funcionado en un sentido similar aunque con un efecto menos intenso- al de los jóvenes de los 80 , puesto que están más interesados en comparación con sus mayores de lo que cabría esperar. Su sensibilidad a los sucesos de naturaleza política y un contexto político capaz de atraer su atención podrían ser las principales explicaciones de este fenómeno. En efecto, en los últimos años se han sucedido varias elecciones excepcionales, aunque parece que el efecto de éstas ha sido más acusado entre los adultos maduros.

Por otra parte, la política cotidiana podría también ser relevante. Sería interesante comprobar si las campañas electorales y las políticas públicas en ciertos años, como 2008, han estado más orientadas a los jóvenes que en otros momentos, de manera que hubieran podido potenciar su interés. Esto podría explicar una parte del fenómeno, ya que en enero de 2008 se aprobó la Renta Básica de Emancipación, que ha sido solicitada por más de 350.000 jóvenes hasta abril de 2010. Esta medida, uno de los pilares de la campaña electoral del PSOE en 2008, podría haber atraído la atención de los jóvenes hacia lo público de una manera similar a como lo hizo la movilización estudiantil entre 1986 y 1987. La misma provocó un gran número de multitudinarias 
manifestaciones y huelgas de alumnos de enseñanza media y universitaria, con las que se solidarizaron los sindicatos obreros. No podemos descartar que el grado de movilización de los sindicatos y asociaciones de estudiantes interaccione con la promulgación de leyes orgánicas sobre la enseñanza y otras normas de carácter general en materia de juventud, provocando un mayor interés por lo público entre los jóvenes. Sin embargo, un análisis más detallado del papel del contexto sobre actitudes como el interés por la política y las interacciones de dicho contexto con características de la población como la edad merecerían un estudio aparte; igual que la comprobación del posible impacto que la crisis económica declarada a partir de 2008 pueda estar causando sobre el interés por la política de jóvenes y adultos. En definitiva, tal vez en España se esté perdiendo la ocasión de superar síndromes como el desapego y la desafección políticas, puesto que el patrón parece ser, en el mejor de los casos, de reproducción cultural. Pero en ningún caso puede decirse que esos síndromes estén agravándose como consecuencia de los niveles de desinterés político entre los jóvenes.

\section{RefERENCIAS BibLIOgRÁFíCAS}

Alcoceba, J. A. y G. Matheus. 2010. El discurso mediático sobre los jóvenes en España. Anuario Electrónico de Estudios en Comunicación Social "Disertaciones" 3 (1), Artículo 11. Disponible en: http://erevistas.saber.ula.ve/index.php/Disertaciones/

Almond, G. A. y S. Verba. 1963. The Civic Culture: Political attitudes and democracy in five nations. Princeton, N. J.: Princeton University Press.

Anduiza, E. 2001. Actitudes, valores y comportamientos políticos de los jóvenes españoles y europeos. Madrid: Injuve.

Anduiza, E. y A. Bosch. 2004. Comportamiento político y electoral. Barcelona: Ariel.

Barnes, S y M. Kaase. 1979. Political Action: Mass participation in Five Western Democracies. Beverly Hills: Sage.

Bonet, E., I. Martín y J. Ramón Montero. 2006. "Actitudes políticas de los españoles". Pp.105-132 en Ciudadanos, Asociaciones y Participación Política en España, coordinado por Joan Font, José Ramón Montero y Mariano Torcal. Madrid: Centro de Investigaciones Sociológicas.

Brady, H. 1999. "Political Participation". Pp. 735-800 en Measures of Political attitudes, edited by John P. Robinson, Phillip R. Shaver y Lawrence S. Wrihtsman. San Diego: Academic Press.

Caínzos, M. 2006. "Participación de los jóvenes españoles en manifestaciones. Comparación con los jóvenes europeos y análisis de sus determinantes". Revista de Estudios de Juventud 75:121-154.

Campbell, A. 1962. "The Passive Citizen." Acta Sociológica, VI (fasc.1-2):9-21.

Delli Carpini, M. X. y S. Keeter. 1996. What Americans Know About Politics and Why It Matters. New Haven y Londres: Yale University Press. 
Durán Muñoz, R. 2007. "La democracia de nuestros mayores. Compromiso cívico y envejecimiento en España", Revista de Investigaciones Políticas y Sociológicas 6:91-105.

Ferrer i Fons, M. 2006. "Jóvenes, participación y actitudes políticas en España, ¿son realmente tan diferentes?", Revista de Estudios de Juventud 75:195-206.

Fiske, S. y S.Taylor. 1991. Social Cognition. Nueva York: McGraw-Hill.

Franklin, M. 2003. "Electoral Competitiveness and Turnout: How voters React to the Changing Character of Elections". Artículo presentado en el European Consortium for Political Research Joint Sessions Workshops. Consulta 1/12/2008 (http://www2.trincoll.edu/ mfrankli/mpsa_proceeding_ 24467. pdf).

Franklin, M. 2004. "Electoral Competitiveness and Turnout: How Voters React to the Changing Character of Elections." Presentado a la reunión anual de la Midwest Political Science Association, Chicago,IL.(http://www.allacademic.com//meta/p_mla_apa_research_citation/0/8/2/4/8/ pages82480/p82480-1.php).

Galais, C. 2008. ¿Socialización o contexto? La implicación política subjetiva de los españoles (19852006). Tesis doctoral defendida el 8 de noviembre de 2008, Departament de Ciències Politiques i Socials, Universitat Pompeu Fabra, Barcelona.

García Albacete, G. 2006. "Jóvenes españoles y política. Análisis y reflexión metodológica a partir de las encuestas del CIS." Informe correspondiente a la Ayuda a la Investigación Sociológica presentado al Centro de Investigaciones Sociológicas. Madrid.

García Albacete, M. Gemma e Irene Martín Cortés. 2010. "La participación política de los jóvenes españoles en perspectiva comparada." Pp. 201-236 en La ciudadanía europea en el siglo XXI. Estudio comparado de sus actitudes, opinión pública y comportamiento políticos, editado por M. Torcal. Madrid: CIS.

García Ibacete, G. 2008. “¿Apatía política? Evolución de la implicación de la juventud española desde los años 80." Revista de Estudios de Juventud 81:133-159.

Goerres, A. 2007. "Why are Older People more Likely to Vote? The Impact of Ageing on Electoral Turnout across Europe." British Journal of Politics and International Relations 9:90-121.

González Anleo, J. 2005. “Jóvenes y valores cívico-políticos.” Educación y Futuro 13:59-70.

Gunther, R., J. R. Montero y J. Botella. 2004. Democracy in Modern Spain. New Haven: Yale University Press.

Henn, M., M. Weinstein y S. Forrest. 2005. "Uninterested Youth? Young People's Attitudes towards Party Politics in Britain." Political Studies 53:556-578.

Inglehart, R. 1990. Culture Shift in Advanced Industrial Societies. Princenton: Princeton University Press. Princeton.

Injuve. 2003. Percepción generacional, valores y actitudes, asociacionismo y participación. Sondeo de opinión y situación de la gente joven. Madrid: Injuve. 
Injuve. 2005. Percepción generacional, valores y actitudes, asociacionismo y participación. Sondeo de opinión y situación de la gente joven. Madrid: Injuve.

Jackman, R. W. 1987. "Political Institutions and Voter Turnout in the Industrial Democracies." American Political Science Review 81:405-424.

Jiménez, M. 2006. “El movimiento por la justicia global: una indagación sobre las aprobaciones de una nueva generación contestataria." Revista de Estudios de Juventud 75:29-42.

Justel, M. 1992. "Edad y cultura política." Revista Española de Investigaciones Sociológicas 58:57-96.

Marsh, D., T. O'Toole and S. Jones. 2007. Young People and Politics in the UK: Apathy or Alienation. New York: Palgrave.

Martin, I. 2004. Los orígenes y significados del interés por la política en dos nuevas democracias: España y Grecia. Madrid: Instituto Juan March.

Martín, I. 2005. "Interés por la política y desapego político". Pp. 63-82 en España: Política y Sociedad en perspectiva comparada. Un análisis de la primera ola de la Encuesta Social Europea. editado por M. Torcal, L. Morales y S. Pérez-Nievas. Valencia: Tirant lo Blanch.

Mateos Díaz, A. 2004. "Una aproximación a las actitudes políticas de los españoles mediante una estructura dimensional inductiva." Revista de Estudios Socio-Jurídicos 6:90-116.

Montero, J. R., R. Gunther y M. Torcal. 1998. "Actitudes políticas de los españoles hacia la democracia: descontento, legitimidad y desafección." Revista Española de Investigaciones Sociológicas 83:9-49.

Morales, L. 2003. "Ever less engaged citizens? Associational membership and political participation in Spain." Working Paper 20, ICPS.

Morales, L. 2005. "¿Existe una crisis participativa? La evolución de la participación política y el asociacionismo en España." Revista Española de Ciencia Política 13: 51-87.

Morales, L., F. Mota y S. Pérez-Nievas. 2006. "La participación en asociaciones: factores individuales". Pp. 157-182 en Ciudadanos, asociaciones y participación política en España, coordinado por J. R. Montero, J. Font y M. Torcal. Madrid: Centro de Investigaciones Sociológicas.

Morán, M. L. y J. Benedicto. 2008. "Los jóvenes como actores sociales y políticos en la sociedad global". Pensamiento iberoamericano 3: 139-164.

Nie, N. H., J. Junn y K. Stehlik-Barry. 1996. Education and Democratic Citizenship in America. Chicago: University of Chicago Press.

Norris, P. 2002. Democratic Phoenix. Reinventing Political Activism. Cambridge: Cambridge University Press.

Plutzer, E. 2002. "Becoming a Habitual Voter: Inertia, Resources, and Growth in Young Adulthood." American Political Science Review 96:41-55.

Pollack, D. y W. Mishler. 2003. "On Culture, Thick and Thin: Toward a Neo-Cultural Synthesis". Pp. 237256 en Political Culture in Post-Communist Europe. Attitudes in New Democracies, edited by Detlef Pollack, Jörg Jacobs, Olaf Müller and Gert Pickel. Aldershot: Ashgate. 
Putnam, R. D. 2000. Bowling Alone. The collapse and revival of American community. Nueva York: Simon and Schuster.

Putnam, R. D., ed. 2002. Democracies in Flux: The Evolution of Social Capital in Contemporary Society. Nueva York: Oxford University Press.

Rosenstone, D. y J. M. Hansen. 1993. Mobilization, Participation, and Democracy in America. Nueva York: MacMillan.

Sapiro, V. 1994. "Political socialization during adulthood: clarifying the political time of our lives." Pp. 197-223 en Micropolitics: New Directions in Political Psychology, edited by M. X. Delli Carpini, L. Huddy and R. Y. Shapiro. Greenwich, CT: JAI Press.

Schreiber, D. y O. García Luengo. 2004. "¿Malestar o círculo virtuoso? Una primera aproximación empírica a la exposición mediática y el compromiso político en España y Alemania", Politica y Sociedad 41:131-143.

Torcal, Mariano. 2006. "Political Disaffection and Democratization History in New Democracies." Pp. 157-189 en Political Disaffection in Contemporary Democracies: Social Capital, Institutions and Politics, editado por Mariano Torcal y José Ramón Montero. London: Routledge.

Van Deth, J. W. 1990. "Interest in Politics". Pp. en Continuities In Political Action. A Longitudinal Study of Political Orientations in Three Western Democracies, edited by J. W. Jennings y M. K. Van Deth. Berlin: Walter de Gruyter.

Verba, S., K. Schlozman y H. Brady. 1995. Voice and Equality: Civic Voluntarism in American Politics. Cambridge: Harvard University Press.

Visser, P. S y J. A. Krosnick. 1998. "Development of Attitude Strength over the life cycle: surge and decline." Journal of Personality and Social Psychology 75:1389-1410.

Weatherford, M. S. 1991. "Mapping the Ties that Bind: Legitimacy, representation and Alienation." Western Political Quarterly 44:251-76.

Zaller, J.R. 1992. The Nature and Origins of Mass Opinion. Cambridge: Cambridge University Press.

CAROL GALAIS es doctora y licenciada en Ciencias Políticas por la Universitat Pompeu Fabra. Actualmente investigadora de la Universitat Autònoma de Barcelona, participa en distintos proyectos relacionados con la participación ciudadana en la administración local, la formación y cambio de las actitudes políticas y las causas de la abstención y el voto. 